Revista de la Facultad de Medicina de la Universidad de lberoamérica
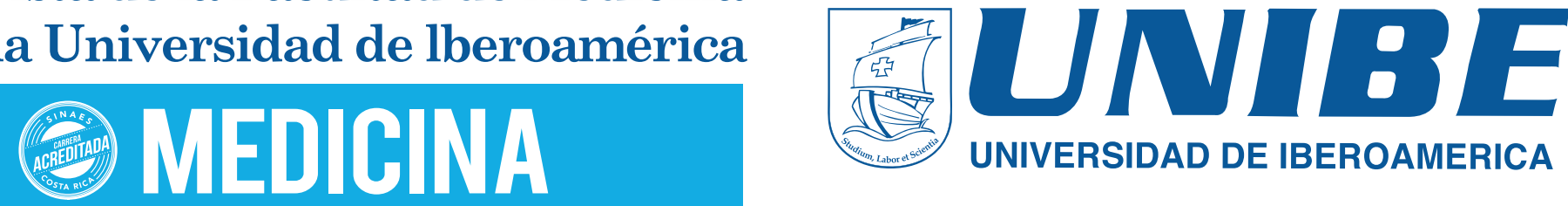

\title{
Artículo
}

\section{Revisión de Farmacología de Urgencias ¿Cómo revertir el efecto de medicamentos anti-tromboticos en sangrado grave?}

Dr. Juan Ignacio Padilla Cuadra

1. Vicedecano de Medicina

Correspondencia con: Dr, Juan Ignacio Padilla Cuadra correo electrónico: revistamedicina@unibe.ac.cr

\section{Resumen}

El uso frecuente de fármacos con efectos antitrombóticos obliga al médico encargado del servicio de emergencias a conocer exhaustivamente su farmacología y muy especialmente como revertir sus

efectos. Cuando un enfermo se presenta con un sangrado que puede estar relacionado con algún medicamento que afecta la hemostasia es conveniente conocer el uso de antídotos específicos, factores de coagulación y algunos hemoderivados.

\section{Abstract}

Common use of antithrombotic agents makes mandatory that emergency physician know its pharmacology and reversal approach. When a patient under the effect of any type of antithrombotic agent, use of antidotes and hemoderived may be required.

\section{Introducción}

El uso frecuente de fármacos con efectos antitrombóticos obliga al médico encargado del servicio de emergencias a conocer exhaustivamente su farmacología y muy especialmente como revertir sus efectos. Cuando un enfermo se presenta con un sangrado que puede estar relacionado con algún medicamento que afecta la hemostasia es conveniente conocer el uso de antídotos específicos, factores de coagulación y algunos hemoderivados. En esta revisión, se presentan casos clínicos que ilustran este tipo de situaciones y se repasa las acciones pertinentes para su manejo.

\section{Caso 1.}

Femenina 80 años, con antecedente de hipertensión arterial, diabetes mellitus y dislipidemia ingresa al servicio de emergencias. 


\section{(2) MEDICINA}

Según refiere su hija, la paciente toma tratamiento oral que incluye irbesartan, metformina y lovastatina.

Recientemente, su médico de cabecera la ha indicado aspirina y clopidrogel debido a que presentó un episodio de isquemia cerebral transitoria asociada a disartria y disminución de fuerza en hemicuerpo derecho. Ahora es traida por presenta caída desde su propia altura asociada a deterioro de conciencia. Se realiza tomografía de cráneo sin contraste (Figura 1).

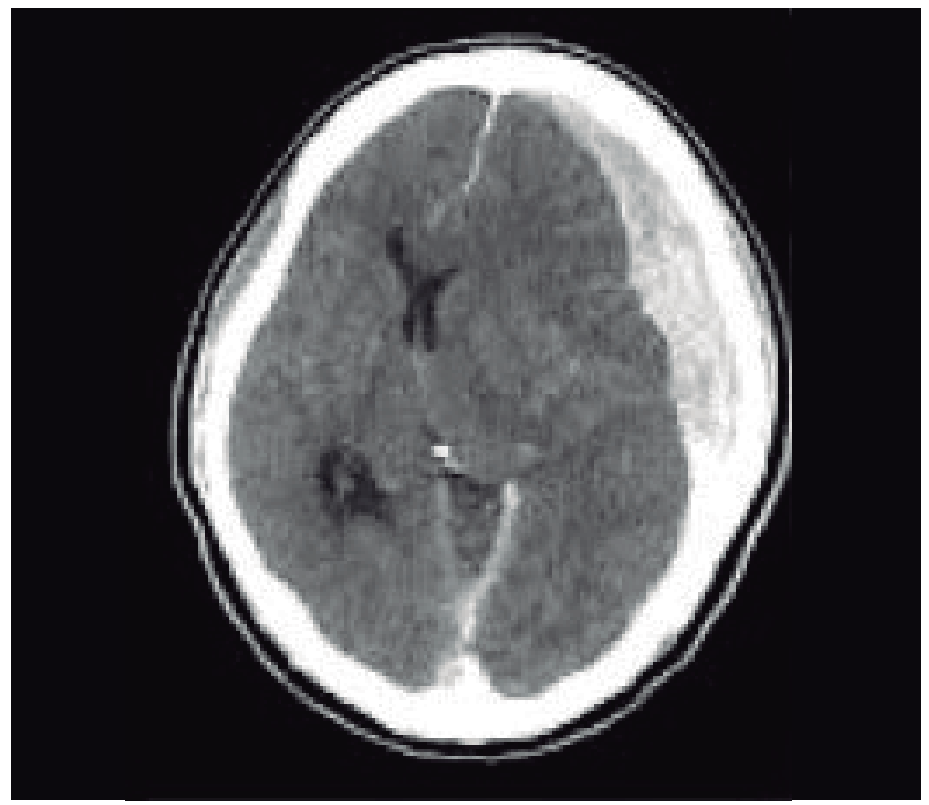

Figura 1. Hematoma subdural izquierdo

Es valorada por neurocirujano quien indica preparar para sala de operaciones ya que su Puntaje de Glasgow ha disminuido y presenta anisocoria.

\section{¿Cómo se revierte el efecto de los medicamentos que la paciente recibe para evitar sangrado transoperatorio?}

\section{Caso 2.}

Masculino 35 años, fue intervenido hace 6 meses para reemplazo mitral mecánico el cual transcurrió sin complicaciones. Egresa con tratamiento con Warfarina. Labora como carpintero y aunque se le advirtió que no debería exponerse a accidentes que le causaran heridas, mientras cortaba varias tablas con sierra de amputa parte de la mano izquierda.

Ingresa al servicio de emergencias con sangrado masivo por el sitio de amputación y por disminución importante de la presión arterial. Los exámenes de laboratorio muestran hemoglobina en $5 \mathrm{gr} / \mathrm{dl}$, tiempo de protrombina en $25 \%$, INR 6 y tiempo de tromboplastina en 30 segundos. El cirujano de trauma le prepara para reparar la lesión vascular y le pregunta a usted como puede revertirse la anticoagulación del paciente.

\section{Caso 3}

Femenina 60 años, conocida portadora de hipertensión, cursa con episodios de disminución de fuerza en hemicuerpo derecho y dificultad para articular palabra. La paciente tiene antecedente de trombosis venosa profunda y está siendo tratada con rivaroxabán (Xarelto ${ }^{\circledR}$ ). Al examen físico, es evidente una hemiplejia Facio-braquio-crural derecha y afasia. Se realiza tomografía de cráneo que muestra sangrado a nivel de ganglios basales en lado izquierdo.

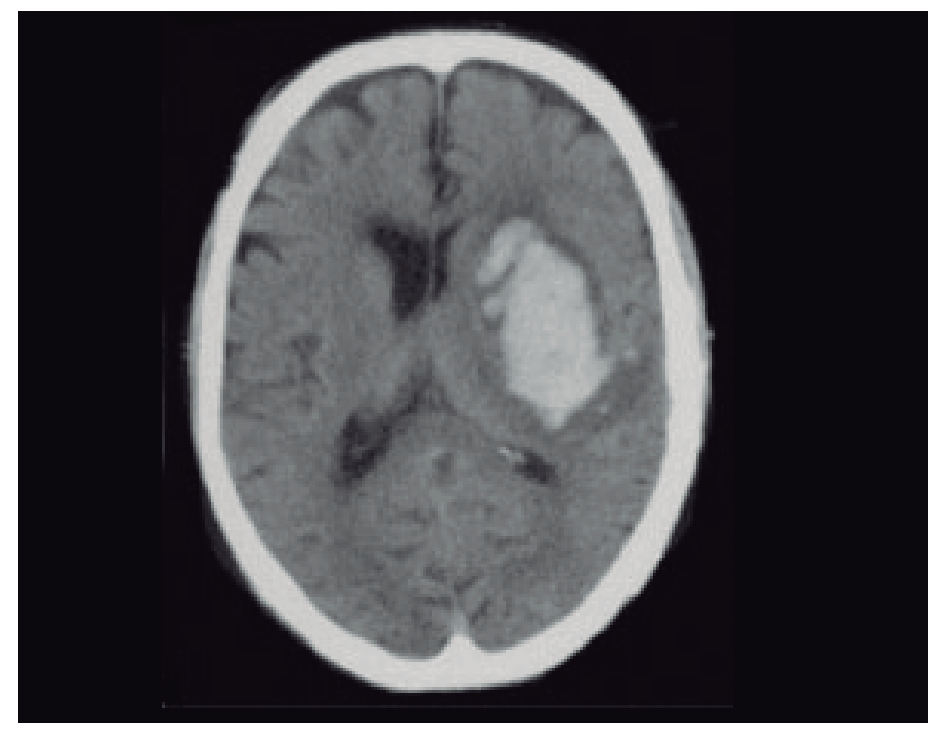

Figura 2. Hemorragia intraparenquimatosa en ganglios basales, lado izquierdo. 
Los exámenes de laboratorio muestran tiempo de protrombina, INR y tiempo de tromboplastina en rangos normales, por lo que el médico de emergencias considera que no es necesario ningún manejo específico. Dos horas después la enferma deteriora su estado de conciencia hasta un puntaje de Glasgow menor a 8 , se decide intubación orotraqueal y repetir la tomografía de cráneo. El nuevo estudio muestra expansión del sangrado original y desviación marcada de la línea media.

En este momento paciente desarrolla midriasis izquierda. ¿Cómo revierte el efecto del agente antitrombótico para poder llevar a sala de operaciones para evacuar el hematoma intraparenquimatoso?

\section{Caso 4.}

Femenina 45 años, portadora de miomatosis uterina. Se programa para cirugía de histerectomía la cual se realiza con diversas dificultades técnicas por múltiples adherencias. En consecuencias, cursa un periodo postoperatorio con severo dolor y esto limita que se pueda movilizar tempranamente. En el postoperatorio 4, presenta episodio de disnea súbito asociado a dolor torácico en hemitórax derecho.

Se realiza estudio de angiotomografía de tórax que demuestra embolismo pulmonar. Se inicia infusión de heparina a razón de 2000 unidades por hora. Al día siguiente, paciente cursa hipotensa con dolor abdominal. Luce pálida y hay franca resistencia a la palpación. Se realiza ultrasonido de abdomen que muestra líquido libre intraabdominal compatible con hemoperitoneo.

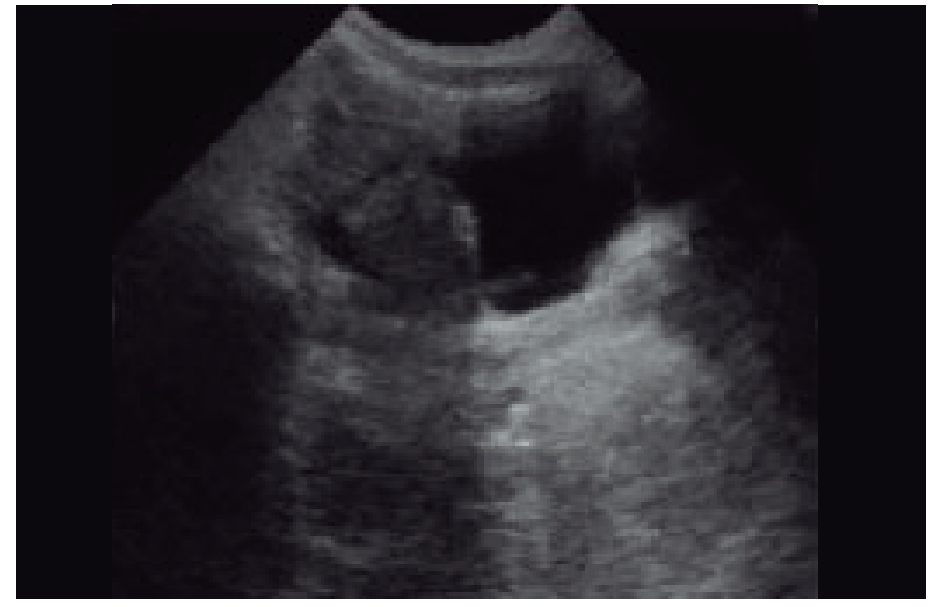

Figura 3. Ultrasonido mostrando líquido libre en pelvis

La hemoglobina es $6.5 \mathrm{gr} / \mathrm{dl}$ (anterior en 10 $\mathrm{gr} / \mathrm{dl}$ ), y el tiempo de tromboplastina es mayor a 200 segundos. El ginecobstetra sospecha sangrado

interno en el sitio de la histerectomía. ¿Qué sugiere debe realizarse para llevar a la paciente a sala de operaciones en una condición hemostática óptima?

\section{Discusión}

Durante situaciones de urgencia asociadas a sangrado que pone en riesgo la vida en pacientes que reciben antitrombóticos deben tenerse en cuenta las siguientes interrogantes: ¿Es necesario revertir de inmediato el efecto del agente?

\section{¿Qué riesgo conlleva la reversión del agente?}

¿Existe un antídoto o acción específica que revierta el agente?

En el caso 1, el uso de dos antiplaquetarios con mecanismos de acción distintos obliga a la reversión de su efecto previo a una intervención neuroquirúrgica. Cuando la intervención de puede diferir, y no es urgente, se puede suspender el medicamento y esperar 5-7 días para que se revierta el efecto antiplaquetario. $\mathrm{Si}$ se necesita revertir el efecto de inmediato, se deben transfundir plaquetas. 
En general se acepta que debe transfundir 10 unidades de plaquetas lo cual se denomina pool. Es importante recordar que, aunque el conteo plaquetario del paciente sea normal esto no indica nada, ya que están inhibidas por el uso de los antiplaquetarios.

En el caso 2, la utilización de anticoagulantes orales tipo cumarínicos o Warfarina es común en el manejo de paciente con válvulas cardiacas protésicas mecánicas. En ausencia de este fármaco, el enfermo estaría en riesgo de trombosis a nivel de la válvula, fenómenos embólicos e incluso disfunción de la misma. La Warfarina deriva su nombre de descubrimiento por parte de estudiantes de Wisconsin, EUA, identificados como Wisconsin Alumni Research Foundation (WARF). Su mecanismo de acción radica en la inhibición de factores de coagulación II, VII, IX y X, proteína C y S, todos ellos vitamina $\mathrm{K}$ dependiente. $\mathrm{Su}$ efecto se cuantifica mediante la medición del tiempo de protrombina.

No obstante, este parámetro puede variar según el reactivo utilizado. Por tanto, se creó un índice universal denominado International Normalized Ratio o INR. Para efectos prácticos, el rango de anticoagulación buscado suele ser de INR entre 2-3.

Para revertir el efecto de la Warfarina, obviamente el primer paso es la suspensión del medicamento, y posteriormente la administración de algunos o varios de los siguientes:

\section{a. Vitamina K endovenosa \\ b. Plasma fresco congelado \\ c. Complejo Protrombínico}

Cada uno de los anteriores tiene un efecto diferente siendo la reversión casi inmediata cuando se usa complejo protrombínico y de hasta 2-4 horas cuando se usa vitamina K. Debe recordarse que, si hay una válvula mecánica, la reversión puede causar trombosis de la misma. La respuesta a la reversión de Warfarina tiene como meta un INR de 1.7 o menos, sino se puede repetir.

Recientemente, se han introducido nuevos anticoagulantes orales (NACOs) cuyo efecto es diferente a la inhibición de los factores de coagulación vitamina $\mathrm{K}$ dependientes. Son ejemplos de estos:

- Pradaxa ${ }^{\circledR}$ (dabigatrán),

- Xarelto ${ }^{\circledR}$ (rivaroxabán)

- Eliquis ${ }^{\circledR}$ (apixabán)

- Lixiana ${ }^{\circledR}$ (edoxabán)

En la actualidad, en nuestro medio, no se cuenta con antídotos específicos para revertir el efecto de estos medicamentos. Según la urgencia de la reversión las opciones son:

Como de revierte el efecto de la Warfarina:

- Vitamina K 10 mg iv +

- Complejo protrombínico (4 factores): 25 a 50 UI por $\mathrm{kg}$ iv en $20 \mathrm{~min}$

Regla General

- Si INR 1.7 a casi 4: $25 \mathrm{u} / \mathrm{kg}$

- Si INR 4-6: $35 \mathrm{U} / \mathrm{kg}$

- Si INR mayor a 6: $50 \mathrm{U} / \mathrm{kg}$

Si no disponible el complejo:

- Plasma fresco congelado 10-15 ml por kg

1. Determine tiempo de última dosis, si dentro de 2 horas, administrar carbón activado 50 gramos vo o sonda 
2. En presencia de deterioro del aclaramiento renal, el efecto puede prolongarse.

3. En caso de necesidad urgente de disminuir el efecto de estos agentes, se puede utilizar la administración de complejo protrombínico razón de

4. Complejo protrombínico (4 50 UI por kilogramo iv (Nivel de evidencia bajo)

Es en extremo importante, recordar que dado el mecanismo de acción de estos agentes, su acción no modifica el INR y por ende este parámetro no es útil para valorar su reversión. El caso 4, muestra un estado excesivo de sobre anticoagulación con heparina.

En general, la heparina no fraccionada se utiliza mediante infusión continua para lograr niveles de anticoagulación que se miden mediante el tiempo de tromboplastina (TPT). En general, la meta es su prolongación a un rango de 1.5 a 2 veces el basal del paciente.

El antídoto de la heparina es el sulfato de protamina en donde cada $\mathrm{mg}$ de este medicamento revierte 500 unidades de heparina. Se suele administrar una dosis máxima de $\mathrm{xxx} \mathrm{mg}$ endovenoso por infusión lenta. La protamina puede causar hipotensión y en algunos casos reacciones alérgicas en pacientes con antecedente de alergia a pescado. La meta es llevar el tiempo de tromboplastina a niveles normales (30 a 35 segundos).

\section{Conclusión}

El uso frecuente y el crecimiento en la variedad de agente antitrombóticos obliga al médico a conocer las opciones para revertir su efecto.

Esto es primordial al momento de manejar un paciente con un sangrado que amenaza la vida.

\section{Lecturas recomendadas:} 1. Frontera JA, et al. Guideline for Reversal of Antithrombotics in Intracranial Hemorrhage: A Statement for Healthcare Professionals from the Neurocritical Care Society and Society of Critical Care Medicine. Neurocrit Care. 2016;24(1):6-46.

2. Makris $\mathrm{M}$, et al. Guideline on the management of bleeding in patients on antithrombotic agents. $\mathrm{Br} J$ Haematol. 2013;160(1):35-46.

Reversión de la Heparina no fraccionada:

- Sulfato de protamina $1 \mathrm{mg}$ iv por cada $100 \mathrm{U}$ de heparina suministradas en las últimas 2 horas. Máximo $50 \mathrm{mg}$.

- Meta: normalizar TPT

- Si persiste TPT prolongado, considere repetir con dosis $0.5 \mathrm{mg}$ iv por cada 100 unidades de heparina suministradas en las últimas 2 horas

- Recuerde: Cada ampolla de Protamina contiene $25 \mathrm{mg}$ 